\title{
Isolation of actinomycetes from cervical specimens
}

\author{
RM TRAYNOR, D PARRATT, HELEN LD DUGUID,* ID DUNCAN $\dagger$ \\ From the Department of Bacteriology, Ninewells Hospital, *Department of Pathology, Cytology Unit, Royal \\ Infirmary, and the †Department of Obstetrics and Gynaecology, Ninewells Hospital, Dundee
}

SUMMARY Cervical smears from 30 women were examined. Fifteen of these had actinomycetes visible by direct smear examination and the organisms were isolated in 13. Of the remainder, all had negative smears and culture was similarly negative in 12 , whilst in 3 , the cultures were either positive (1) or suspicious (2).

During the last ten years actinomycetes have increasingly been recognised in cervical smears of women using intrauterine contraceptive devices (IUCD). The identification of these actinomycetes has mainly been based on morphological findings. ${ }^{1-3}$ However, Bhagavan and Gupta ${ }^{4}$ isolated four strains of actinomycetes from 11 cases $(36.3 \%)$ taken from a series of 36 women diagnosed by Papanicolaou stain. Two of these isolates were identified as Actinomyces israelii using fluorescein-labelled antisera; the other two isolates were not ascribed to a species.

It is well documented that actinomycetes are difficult to obtain from a mixed culture ${ }^{5-7}$ and that there are at least six different strains of actinomycetes which can produce actinomycotic infections. ${ }^{8}$ Therefore a cultural method which improves the isolation rate would be of benefit in diagnosis and an aid towards precise identification which in turn would assist the investigation of cervical infection.

In a previous retrospective study we showed that actinomycetes were present in 42 of $293(14 \%)$ IUCD users and that most of these women were using all-plastic devices. ${ }^{9}$ In a continuing study, women found to have organisms suggestive of actinomycetes in routine cervical smears are being recalled to a special clinic. As part of this study attempts are made to culture the organisms from cervical swabs and from removed devices.

\section{Patients and methods}

Thirty women in two groups (A and B) were examined at a special clinic. In group $A$ cases, referral was because actinomyces-like-organisms (ALOs) had been seen or suspected in cervical smears stained by Papanicolaou's method at a previous examination,

Accepted for publication 14 January 1981 whilst group B women were randomly selected from those attending a family planning clinic. Two of group B were shown on first examination to have cervical smears positive for ALOs and these were transferred thereafter to group A. In group A cases, the second examination was within a 2-5 month period of the first and all women still had an IUCD in situ. Cervical smears and swabs were taken from the external os in these women and in six cases, the IUCD was removed, and studied bacteriologically.

The cervical smears were Gram-stained and examined for leucocytes, ALOs and microcolonies of actinomycetes. Within two hours of sampling, bacteriological examination was made on the swabs and selected beads of pus from the device.

\section{CULTURE METHOD}

A primary inoculum was made by soaking the swabs or emulsifying selected beads of pus from the device in $5 \mathrm{ml}$ thioglycollate broth (BBL). From this primary inoculum a series of tenfold dilutions was made in thioglycollate broth $\left(10^{-1}\right.$ to $\left.10^{-4}\right) \cdot 0 \cdot 1 \mathrm{ml}$ of each dilution was inoculated in duplicate on two $10 \%$ horse-blood agar plates (Columbia agar base, Oxoid), and spread to produce a lawn. A concentration of $2.5 \mathrm{mg} / \mathrm{l}$ of metronidazole was incorporated in half the plates to reduce the growth of anaerobic flora and allow the actinomyces to grow. Actinomycetes are relatively resistant to this concentration of metronidazole. All plates were incubated anaerobically at $37^{\circ} \mathrm{C}$ in a McIntosh-Fildes jar in an atmosphere of $90 \% \mathrm{H}_{2}, 10 \% \mathrm{CO}_{2}$ and examined after 4, 10, and 14 days. All different colony types were Gram-stained and any Gram-positive bacilli, whether branching or not, were re-examined by the immunofluorescent technique.

IMMUNOFLUORESCENT METHOD

The immunofluorescent method was an indirect 914 
method; rabbit antiactinomycete antiserum was used in the first stage and fluorescein labelled sheep antirabbit globulin in the second stage. The first antibody was raised by injecting an animal with approximately $10^{8}$ Actinomyces israelii (NCTC 8439) made up in water-in-oil emulsion containing Mycobacterium tuberculosis (complete adjuvant). An injection $(0.4 \mathrm{ml})$ was given intramuscularly to each gluteal region and $0.2 \mathrm{ml}$ subcutaneously at the nape of the neck. After six weeks, intravenous booster injections of a saline suspension $\left(10^{8} \mathrm{ml}\right)$ of the same organism were started with $0.3 \mathrm{ml}$ which was increased by $0 \cdot 1 \mathrm{ml}$ per week until the animal was receiving $1.0 \mathrm{ml}$. After a further week the animal was bled to obtain the antiserum which was tested by the immunofluorescent method. The method used was standard, involving incubation periods of $30 \mathrm{~min}$ at each stage and using phosphate-buffered saline (pH 7.4) as the washing solution. Initial studies showed that the antiserum did not react with known diphtheroids, lactobacilli, coliforms or Gram-positive cocci, and that it produced strong fluorescence with Actino israelii to a dilution of $1 / 50$. The antiserum also gave strong fluorescence with Actino viscosus (NCTC 10951), Actino odontolyticus (NCTC 9931) and Actino naeslundii (NCTC 10301). No fluorescence was obtained with Actino bovis (NCTC 4500).

\section{Results}

No actinomycetes were seen in any cultures at the 4-day examination. Of those subsequently found positive, the majority were recognisable as actinomycetes after 10 days incubation, and a few required up to 14 days to become obvious. The results for all patients are shown in the Table.

In group A, fifteen smears were positive for ALOs and in 13 of these, actinomycetes were isolated. One of the others was culture-negative and the remaining one produced a "suspicious" organism-that is, an organism morphologically similar to an actinomycete, but giving a weak immunofluorescent reaction. For three smear-negative women, two suspicious cultures and one positive culture were obtained. In the majority of those specimens from which actinomycetes were cultured, the metronidazole-blood agars produced the growth, and ordinary blood agar culture was unsuccessful because of overgrowth by anaerobic organisms.

All smears and cultures were negative in group B.

Six removed devices were examined, and produced five positive and one suspicious culture, in each case correlating exactly with results from the respective cervical swabs.

\section{Discussion}

Actinomycetes have been recognised in cervical smears in women using IUCDs. ${ }^{2}{ }^{410-13}$ In a previous retrospective study we showed that actinomycetes were present in cervical smears in 42 of 293 IUCD users $(14.3 \%)$, and that most of these women were using plastic devices. ${ }^{9}$ As part of the present study, attempts were made to culture the organism from

Results of smear examination and culture results for all patients

\begin{tabular}{|c|c|c|c|c|c|c|c|}
\hline \multicolumn{4}{|c|}{ Group $A$} & \multicolumn{4}{|c|}{ Group $B$} \\
\hline No & Smear result & $\begin{array}{l}\text { Culture of cervical } \\
\text { swabs }\end{array}$ & Culture of IUCD & $N o$ & Smear result & $\begin{array}{l}\text { Culture of cervical } \\
\text { swabs }\end{array}$ & Culture of IUCD \\
\hline 1 & + & + & + & 1 & - & - & \\
\hline 2 & + & + & + & 2 & - & - & \\
\hline 3 & + & + & & 3 & - & - & \\
\hline 4 & + & + & + & 4 & - & - & \\
\hline 5 & + & + & + & 5 & - & - & \\
\hline 6 & + & susp & & 6 & - & - & \\
\hline 7 & + & + & & 7 & - & - & \\
\hline 8 & + & + & & 8 & - & - & \\
\hline 9 & + & + & + & 9 & - & - & \\
\hline 10 & + & + & & 10 & - & - & \\
\hline 11 & - & susp & & 11 & - & - & \\
\hline 12 & - & + & & 12 & - & - & \\
\hline 13 & + & + & & & & & \\
\hline 14 & + & + & & & & & \\
\hline 15 & + & + & & & & & \\
\hline 16 & + & + & & & & & \\
\hline 17 & - & susp & susp & & & & \\
\hline 18 & + & - & & & & & \\
\hline
\end{tabular}

$+=$ positive cervical smears which showed Gram-positive branching rods, often as microcolonies. Positive cultures were those producing colonies which were subsequently shown to contain Gram-positive branching bacilli and a strong immunofluorescence reaction. susp = "suspicious" cultures which showed small, dry colonies containing Gram-positive bacilli without obvious branching, and which gave weak fluorescence reactions.

$-=$ negative for actinomyces-like-organisms. 
cervical swabs and from removed devices and in view of the success of our methods we have felt that early publication of the results is required.

In this study we have isolated actinomycetes from 13 of 15 women who had positive cervical smears on re-examination. One of the remaining two patients had a suspicious culture-that is, one in which actinomyces-like-organisms were obtained, but we were not sure that they were true actinomycetes. One patient has a positive smear but negative culture. Thus the success of our cultural method is at least $86 \%$ and this compares with other investigations where the results have been negative ${ }^{3}$ and $36 \%{ }^{4}$ In control patients all cervical smears and cultures were negative indicating that with the technique, isolation of actinomycetes from all cervical swabs, regardless of origin, does not occur. However, in the referred patients, one actinomycete and two "suspicious" organisms were isolated where the smears had been negative, indicating perhaps that the cultural method is slightly more sensitive. Where culture from removed devices was carried out the results confirmed the swab results exactly.

It should be noted that culture for other organisms (not only actinomycetes) is being carried out in this study and full details of this aspect will be reported later with the clinical findings. In all cases, however, a heavy mixed anaerobic flora has been obtained regardless of whether actinomycetes have been isolated. We suggest that the success of our method for obtaining the actinomycetes is related to (a) prolonged anaerobic incubation; (b) the use of metronidazole to inhibit other anaerobic flora; (c) the use of Columbia blood agar which supports actinomycetes well, and (d) a dilutional technique which makes identification of small actinomycete colonies much easier.

Confirmation of "suspicious" colonies as actinomycetes is often difficult because these organisms do not always show branching and therefore appear as diphtheroids or lactobacilli. The immunofluorescent method which we have used, whilst not ascribing the organism to a species, does allow a more confident acceptance or rejection of identity of the "suspicious" colonies. For this reason, we have in the study three isolates which although apparently actinomycetes, do not react well with the antisera (known to be reactive with NCTC strains) and do not show branching. These organisms may ultimately be shown to be true actinomycetes but at this stage they must be regarded only as "suspicious" colonies. Thus with the immunofluorescence method, for the majority of isolates, we have been able to be confident of their identity as actinomycetes.

It is possible that the antiserum used for immunofluorescence does not react with all actinomycetes, and further work is required when other strains can be made available. Further, it is likely that suitable absorption of the antiserum may ultimately permit full identification of all organisms by immunofluorescence, although at this stage it is impossible to be confident that all the isolated organisms are Actino israelii, as has been suggested in other studies. ${ }^{11} 14$

We wish to thank our colleagues in the Family Planning Clinics in the Dundee area, and Searle Ltd for financial support.

\section{References}

${ }^{1}$ Hager DW, Majumdar B. Pelvic actinomycosis in women using intrauterine contraceptive devices. Am J Obstet Gynecol 1979;133:60-3.

${ }^{2}$ Schiffer MA, Elguezabal A, Sultana M, Allen MC. Actinomycosis infections associated with intrauterine contraceptive device. Obstet Gynecol 1975;45:67-72.

${ }^{3}$ Schiffer MA, Elguezabal A, Allen MC. Actinomycosis infections associated with intrauterine contraceptive devices and a vaginal pessary. Advances in Planned Parenthood 1978;12:183-92.

${ }^{4}$ Bhagavan BS, Gupta PK. Genital actinomycosis and intrauterine contraceptive devices. Hum Pathol 1978;9: 567-78.

${ }^{5}$ Berardi RS. Abdominal actinomycosis. Surg Gynecol Obstet 1979;149:257-66.

${ }^{6}$ Brown JR. Human actinomycosis; a study of 181 subjects. Hum Pathol 1973;4:319-30.

${ }^{7}$ Weed LA, Baggenstoss AH. Actinomycosis: a pathologic and bacteriologic study of twenty-one fatal cases. Am J Clin Pathol 1949;19:201.

${ }^{8}$ Slack JM. The epidemiology of actinomycosis. In: AlDoory Y, ed. The epidemiology of human mycotic disease. Springfield, Illinois: Charles Thomas, 1976.

${ }^{9}$ Duguid Helen LD, Parratt D, Traynor R. Actinomyceslike-organisms in cervical smears from women using intrauterine contraceptive devices. $B r J$ Med 1980;281 : 534-41.

${ }^{10}$ Gupta PK, Hollander D, Frost JK. Actinomycetes in cervicovaginal smears: an association with IUD usage. Acta Cytol (Baltimore) 1976;20:295-7.

${ }^{11}$ Spence MR, Gupta PK, Frost JK, King TM. Cytologic detection and clinical significance of Actinomyces israelii in women using intrauterine contraceptive devices. Am J Obstet Gynecol 1978;131:295-8.

12 Jones Mary C, Buschmann BD, Dowling EM, Pollock Helen M. The prevalence of actinomycetes-like-organisms found in cervicovaginal smears of 300 IUD wearers. Acta Cytol (Baltimore) $1979 ; 23: 282-6$.

13 Lomax CW, Harbert GW, Thornton WNJ. Actinomycosis of the female genital tract. Obstet Gynecol 1976;48:341-6.

${ }^{14}$ Luff RD, Gupta PK, Spence MD, Frost JK. Pelvic actinomycosis and the intrauterine contraceptive device. Am J Clin Pathol 1978;69:581-6.

Requests for reprints to: Dr D Parratt, Department of Bacteriology, University of Dundee Medical School, Ninewells Hospital, Dundee DD1 9SY, Scotland. 